\title{
Clostridium difficile Infection After Ileostomy Reversal
}

\author{
Ho Seung Kim, Jae Hyun Kang, Han-Gil Kim, Young Hun Kim, Hyeonwoo Bae, Nam Kyu Kim \\ Division of Colorectal Surgery, Department of Surgery, Severance Hospital, Yonsei University College of Medicine, Seoul, Korea
}

Clostridium difficile infection (CDI) after ileostomy reversal is rare, with few reports available in the available literature describing this condition. The diagnosis of CDI after ileostomy reversal is challenging because symptoms such as diarrhea observed in these patients can occur frequently after surgery. However, CDI can be fatal, so early diagnosis and prompt treatment are important. We discuss 2 patients with positive $C$. difficile toxin assay results on stool cultures performed after ileostomy reversal. Clinical progression differed between these patients: one patient who presented with severe CDI and shock was successfully treated following a prolonged intensive care unit stay for the management of vital signs and underwent hemodialysis, while another patient showed symptoms of mild colitis but we could not confirm whether diarrhea was associated with CDI or with the usual postoperative state. To our knowledge, these represent 2 of just a few cases reported in the literature describing CDI after ileostomy reversal.

Keywords: Clostridium difficile infection; Ileostomy reversal; Colonization

\section{INTRODUCTION}

Clostridium difficile infection (CDI) is a major cause of hospitalacquired infection that continues to achieve increasing levels of incidence and severity among hospitalized patients. Symptoms can range from mild diarrhea to fulminant colitis that can result in severe sepsis, toxic megacolon, and multiorgan failure, and this condition is associated with high mortality [1].

Few reports to date in the available literature have described CDI after ileostomy reversal. Reportedly, the incidence of CDI was significantly higher in patients who underwent ileostomy reversal than in patients who underwent other colorectal procedures, including anterior resection, right hemicolectomy, and appendectomy [2]. The pathogenesis of this condition is attributed to mucosal colonization of the unused colon by $C$. difficile, following the creation of a diverting ileostomy. In animal models, this is

Received: Aug 25, 2019 • Revised: Sep 22, 2019 • Accepted: Sep 24, 2019 Correspondence to: Nam Kyu Kim, M.D.

Division of Colorectal Surgery, Department of Surgery, Severance Hospital, Yonsei University College of Medicine, 50-1 Yonsei-ro, Seodaemun-gu, Seoul 03722, Korea

Tel: +82-2-2228-2100, Fax: +82-2-313-8289

E-mail:namkyuk@yuhs.ac

ORCID: https://orcid.org/0000-0003-0639-5632

(C) 2021 The Korean Society of Coloproctology

This is an open-access article distributed under the terms of the Creative Commons Attribution NonCommercial License (https://creativecommons.org/licenses/by-nc/4.0) which permits unrestricted noncommercial use, distribution, and reproduction in any medium, provided the original work is properly cited. associated with changes in the microenvironment secondary to mucosal and muscular atrophy that occur in the defunctioned colon [3].

We report on 2 patients with positive $C$. difficile toxin assay results following stool cultures performed after ileostomy reversal. The clinical progression differed between these patients, although the toxin assay in both cases showed positive results for $C$. difficile.

\section{CASE REPORTS}

\section{Case 1}

A 65-year-old male visited the emergency department with a 1 -day history of abdominal pain and severe diarrhea. His blood pressure was $65 / 50 \mathrm{mmHg}$ and pulse rate was 121 beats/min. Non-contrast-enhanced abdominopelvic computed tomography (CT) revealed edematous, thickened walls of the entire colon with pericolic fat infiltration and fluid collection (Fig. 1). Laboratory tests revealed a significantly elevated white blood cell count $(32,810 / \mu \mathrm{L})$ and serum levels of C-reactive protein $(488 \mathrm{mg} / \mathrm{L})$, procalcitonin $(>100 \mathrm{ng} / \mathrm{mL})$, and creatine $(3.82 \mathrm{mg} / \mathrm{dL})$. Stool toxin assays confirmed CDI. The patient was admitted to the intensive care unit (ICU) owing to unstable vital signs. Conservative management was initiated with the administration of intravenous (IV) antibiotics and oral vancomycin due to a diagnosis of CDIinduced shock.

The patient's history revealed he had undergone laparoscopic ultra-low anterior resection and creation of a diverting ileostomy 


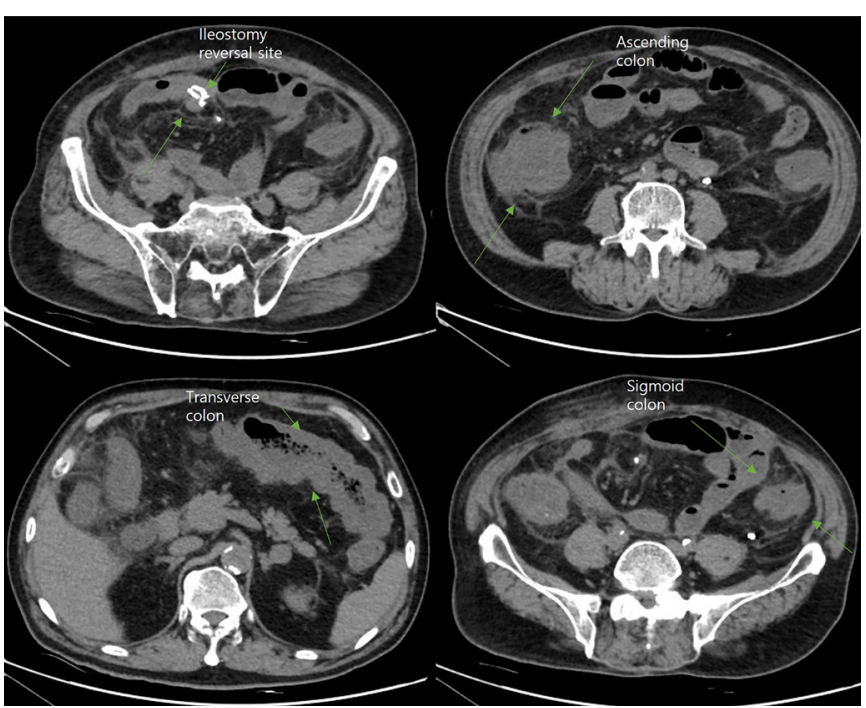

Fig. 1. Non-contrast-enhanced computed tomography scan showing pancolitis.

for rectal cancer after receiving chemoradiation therapy 8 months prior to the current presentation. He also had a past medical history of hypertension and diabetes mellitus. He had undergone elective ileostomy reversal 10 days earlier, receiving a single dose of parenteral cefotetan on the day of surgery and completing a successful surgery without any intraoperative events. His postoperative course was uneventful and he was discharged on the 5th postoperative day with medication for wound pain.

Initially, he received inotropic medication and underwent hemodialysis to support his vital signs, recovering gradually with this treatment. Inotropic agents were tapered completely on the 10th day after admission to the ICU. He could tolerate a soft diet on the 12th day, and hemodialysis was discontinued on the 23rd day following admission to the ICU. Owing to his prolonged ICU stay, he required rehabilitation for muscle weakness and was subsequently discharged 37 days after admission. Vancomycin was used for 14 days.

\section{Case 2}

A 57-year-old male was admitted for ileostomy reversal. He had undergone laparoscopic low anterior resection and creation of a diverting ileostomy for rectal cancer 8 months prior to this presentation. He had no notable past medical history other than he had undergone elective ileostomy reversal. At the time, he had received a single dose of parenteral cefotetan on the day of the operation and undergone successful surgery without any intraoperative events.

Postsurgery, he complained of vomiting and radiography revealed ileus until the fifth postoperative day. His laboratory tests revealed no abnormalities. Watery diarrhea were observed on the sixth postoperative day, and a stool toxin assay confirmed the presence of C. difficile. Conservative management was initiated with the administration of IV antibiotics and oral vancomycin. He could tolerate a soft diet on the ninth postoperative day and was discharged on the 11th postoperative day without any complications. Vancomycin was used for 7 days.

These case reports were approved by the Institutional Review Board of Severance Hospital and the requirement for informed consent was waived (No. 4-2019-0558).

\section{DISCUSSION}

Few case reports in the available literature have described CDI after ileostomy reversal [4-6]. Abe et al. [4] reported a case of severe fulminant CDI-induced colitis occurring 3 days after ileostomy reversal in a patient who died following emergency total colectomy. Fashandi et al. [6] also described fulminant CDI at 3 days after ileostomy reversal in a patient who died on the fifth postoperative day despite emergency total colectomy. In the cases reported by these authors, the patients showed a rapid deterioration in their clinical course beyond the scope of the conventional management algorithm. The patient reported as case 1 in our report showed a similar fulminant course with severe pancolitis and shock. Early CT or colonoscopy is essential for the prompt diagnosis and treatment of pseudomembranous colitis.

The early and accurate diagnosis of CDI based on symptomatic evaluation is challenging in patients undergoing ileostomy reversal. Early symptoms of CDI such as diarrhea might be misdiagnosed as normal and expected findings in patients undergoing ileostomy closure. Almerie et al. [5] described a finding of CDI after ileostomy reversal is similar in a patient who presented with diarrhea, which was suspected to be secondary to causes such as anastomotic leak, iatrogenic bowel injury, or infectious colitis (based on elevated serum levels of inflammatory markers accompanying the diarrhea). Sudden and severe diarrhea accompanied by abdominal pain and elevated serum levels of inflammatory markers (as observed in case 1) should lead to a high index of clinical suspicion for CDI and warrant prompt diagnostic testing.

Hussain et al. [7] described ileostomy effluent stool cultures and protein exotoxin detection in patients who were colonized with $C$. difficile before reversal. In their cohort of 20 patients, no individual presented a positive stool culture or exotoxin test results preoperatively, although stool culture and exotoxin test results were positive postoperatively. This result may be attributed to the fact that $C$. difficile colonizes the unused colon. Shen et al. [8] described patients with fatal CDI-associated pouchitis after ileostomy reversal. These patients underwent total proctocolectomy with ileal pouch-anal anastomosis (IPAA) to treat ulcerative colitis. A study involving 115 patients with ulcerative colitis who underwent IPAA reported that fecal C. difficile toxin testing revealed positive results in $18 \%$ of study participants [9].

Separately, the patient described in case 2 showed symptoms of mild diarrhea, and we could not confirm whether this diarrhea 
was associated with CDI or the usual postoperative state. However, a C. difficile toxin assay showed positive results, indicating $C$. difficile colonization. Previous studies have reported that the risk of subsequent CDI was 5.9-fold higher among patients colonized with toxigenic C. difficile upon hospital admission than in noncolonized patients [10]. Hospitalization within 3 months [10], chemotherapy within the previous 8 weeks [11], antibiotics usage [12] and acid suppression with proton pump inhibitors (PPIs) [13] have been reported as risk factors for $C$. difficile colonizataion. Rubio-Perez et al. [14] reported a significant association between pseudomembranous colitis and delayed ileostomy reversal (of greater than 6 months), with the reported defunctioning time ranging from 9 to 15 months in these patients. Our 2 patients underwent an operation 10 months after the first surgery due to adjuvant chemotherapy. Although neither patient received PPIs, the patient described in case 1 had received chemotherapy 8 weeks earlier, and both chemotherapy and acid suppressants serve as risk factors for colonization and CDI.

A retrospective cohort study performed by Fernandes et al. [15] compared outcomes in patients before and after a change in antimicrobial policy. The authors altered the antibiotic prophylaxis policy used in patients undergoing ileostomy reversal from the administration of three doses of metronidazole plus cefuroxime to a single dose of metronidazole to attempt to promote a reduction in CDI rates. The results showed a 5.8\% decrease in CDI rates, and the postoperative diarrhea rate decreased from $32 \%$ to $12.5 \%$.

We report 2 cases of patients who showed positive C. difficile toxin assay results on stool cultures performed after ileostomy reversal. Despite the low incidence, the clinical presentation of this condition is often indistinguishable from the usual postoperative state; therefore, diagnosis is challenging. Fulminant cases are known to occur; therefore, clinicians must consider this condition in the differential diagnosis, and prompt evaluation is warranted in patients undergoing ileostomy reversal who present with severe diarrhea and abdominal pain. Clinicians should be aware of the risk factors for CDI or colonization and reduce the use of unwarranted antibiotics and PPIs and consider earlier ileostomy reversal where appropriate.

\section{CONFLICT OF INTEREST}

No potential conflict of interest relevant to this article was reported.

\section{REFERENCES}

1. Adams SD, Mercer DW. Fulminant Clostridium difficile colitis. Curr Opin Crit Care 2007;13:450-5.

2. Randall JK, Young BC, Patel G, Fitzgerald A, George BD. Is Clos- tridium difficile infection a particular problem after reversal of ileostomy? Colorectal Dis 2011;13:308-11.

3. Kissmeyer-Nielsen P, Christensen H, Laurberg S. Diverting colostomy induces mucosal and muscular atrophy in rat distal colon. Gut 1994;35:1275-81.

4. Abe I, Kawamura YJ, Sasaki J, Konishi F. Acute fulminant pseudomembranous colitis which developed after ileostomy closure and required emergent total colectomy: a case report. J Med Case Rep 2012;6:130.

5. Almerie MQ, Culverwell A, Krishna J, Mahon C. Chronic inflammation masquerading as an appendiceal tumour with peritoneal metastasis: the challenge of diagnosis and the lessons learnt. BMJ Case Rep 2016;2016:bcr2015213911.

6. Fashandi AZ, Ellis SR, Smith PW, Hallowell PT. Overwhelming recurrent Clostridium difficile infection after reversal of diverting loop ileostomy created for prior fulminant $\mathrm{C}$. difficile colitis. Am Surg 2016;82:e194-5.

7. Hussain ZI, Todd N, Adams S, Stojkovic SG. Prevalence of clostridium difficile in excluded colons. Am Surg 2012;78:408-13.

8. Shen B, Remzi FH, Fazio VW. Fulminant Clostridium difficileassociated pouchitis with a fatal outcome. Nat Rev Gastroenterol Hepatol 2009;6:492-5.

9. Shen BO, Jiang ZD, Fazio VW, Remzi FH, Rodriguez L, Bennett $\mathrm{AE}$, et al. Clostridium difficile infection in patients with ileal pouch-anal anastomosis. Clin Gastroenterol Hepatol 2008;6:7828.

10. Zacharioudakis IM, Zervou FN, Pliakos EE, Ziakas PD, Mylonakis E. Colonization with toxinogenic C. difficile upon hospital admission, and risk of infection: a systematic review and metaanalysis. Am J Gastroenterol 2015;110:381-90.

11. Loo VG, Bourgault AM, Poirier L, Lamothe F, Michaud S, Turgeon N, et al. Host and pathogen factors for Clostridium difficile infection and colonization. N Engl J Med 2011;365:1693-703.

12. Vonberg RP, Kuijper EJ, Wilcox MH, Barbut F, Tull P, Gastmeier P, et al. Infection control measures to limit the spread of Clostridium difficile. Clin Microbiol Infect 2008;14 Suppl 5:2-20.

13. Jump RL, Pultz MJ, Donskey CJ. Vegetative Clostridium difficile survives in room air on moist surfaces and in gastric contents with reduced acidity: a potential mechanism to explain the association between proton pump inhibitors and C. difficile-associated diarrhea? Antimicrob Agents Chemother 2007;51:2883-7.

14. Rubio-Perez I, Leon M, Pastor D, Diaz Dominguez J, Cantero R. Increased postoperative complications after protective ileostomy closure delay: an institutional study. World J Gastrointest Surg 2014;6:169-74.

15. Fernandes R, Robinson P, Rangarajan K, Scott S, Angco L. The role of single-shot metronidazole in the prevention of Clostridium difficile infection following ileostomy reversal surgery. Int J Colorectal Dis 2017;32:733-6. 